\title{
An Improved GM-PHD Approach for Multi Extended Target Tracking with Square-root Cubature Information Kalman Filter
}

Zhe Liu ( $\square$ liuzhe@nuc.edu.cn )

North University of China https://orcid.org/0000-0003-3028-3657

\section{Fengbao Yang}

North University of China

Linna Ji

North University of China

Xiqiang Qu

North University of China

\section{Research}

Keywords: Multi extended targets, Gaussian mixture, probability hypothesis density, square-root cubature information filter

Posted Date: June 22nd, 2021

DOl: https://doi.org/10.21203/rs.3.rs-605645/v1

License: (9) This work is licensed under a Creative Commons Attribution 4.0 International License. Read Full License 


\title{
An Improved Gaussian Mixture Probability Hypothesis Density Approach for Multi Extended Target Tracking with Square-root Cubature Information Kalman Filter
}

\author{
Zhe Liu*, Fengbao Yang, Lina Ji and Xiqiang Qu
}

\author{
"Correspondence: \\ liuzhe@nuc.edu.cn \\ School of Information and \\ Communication Engineering, \\ North University of China, 030051 \\ Taiyuan, China \\ Full list of author information is \\ available at the end of the article
}

\begin{abstract}
The conventional target tracking approaches are presented under the assumption of the point target. In multi extended target scenarios, the tracking performance of these approaches may be greatly decreased. The the Gaussian mixture (GM) implementation of the probability hypothesis density (PHD) (named as the ET-GM-PHD approach) has been presented for applying the GM-PHD approach into extended target tracking. However, it has been proposed under the linear models. In fact, most of targets are moving with nonlinear models. Thus, we, in this paper, present a square-root cubature information filter (SCIF) based ET-GM-PHD approach. To be more specific, we, first, employ the cubature points to predict the mean and the square-root factor of covariance. Then, the information forms of the mean and square-root of covariance has been used to update the mean and covariance of GM component. Meanwhile, we integrate the gating method into our method for saving computational complexity. Owing to the significant tracking performance of the SCIF method, our approach can estimate states and number of multi extended targets in nonlinear scenarios. In addition, we also propose an observation driven method to initiate the birth intensity. As for our method, the conditional probability has been adopted to describe the association between the target and its corresponding observations. With such a probability, the most possible partition, where the estimated targets belong to, can be approximated. Thus, the birth intensity can be estimated by removing the cells associated with the estimated targets. Since we use the estimated targets to initiate the birth intensity, our approach can initiate the birth intensity adaptively. The simulation results prove the effectiveness of our approach.
\end{abstract}

Keywords: Multi extended targets; Gaussian mixture; probability hypothesis density; square-root cubature information filter

\section{Introduction}

Background

Multi extended target tracking refers to estimating the states states (positions, velocities, etc.) and number of extended targets. Most of the traditional methods has been proposed under the point target assumption. As for such an assumption, the target can generate at most one observation. However, for modern radars with high resolutions, the target may locate in several cells of the radar beam. That means, the target may generated more than 
one observation. These targets has been named as the extended targets $[1,2,3]$. Compared with the point target tracking, the associations, such as observation-to-observation and state-to-observation, are rather hard to be achieved. Therefore, the conventional point target based approaches [4, 5], such as the nearest-neighbour Kalman filter (NNKF), multiple hypothesis tracking (MHT), and joint probabilistic data association (JPDA), cannot be effective in such scenarios.

Several approaches have been proposed for solving multi extended target tracking problem. Feldmann et. al [6] applied the random matrix to describe the extent of the extended target. However, it assumes that the extended targets follow the ellipsoidal shape. For tracking targets with non-ellipsoidal shapes, Lan et. al [7] model the extended targets with a group of sub-ellipsoidal extended objects. On this basis, the multiple-model (MM) approach has been proposed for tracking extended targets. Nevertheless, the performance of the estimating accuracy has been dependant on the number of sub-ellipsoidal objects. A Gaussian process model of observations under the probabilistic multi hypothesis tracker (PMHT) framework has been present in [8]. Such a model can process the extended and point-like target seamlessly. However, the associations between states and observations are rather difficult to be achieved. Daniyan et. al [9] presented a multi extended targeting tracking approach using the labeled random finite set. In such an approach, the B-splines and Poisson mixture variational Bayesian are employed to estimate the states of extended targets. Granström et. al [10] modeled the modeled the observations of the extended target as the Poisson process. With such a model, he probability hypothesis density (PHD) filter (named as EPHD) has been proposed in [11]. However, there is no close-form solutions for the EPHD filter [12]. Zhang et. al [13] utilized a group of box particles to convert the multi dimensional integrals into summation of particles. When the number of particles grows large, it may cost great computational complexity. Instead, Granström et. al [14, 15] use a group of Gaussian mixture (GM) component to estimate states and number of extended targets. Such a method has been named as the ET-GM-PHD approach. Since the number of GM components is much smaller the particles, the computational complexity can be greatly saved. For improving the tracking performance of the ET-GM-PHD approach, Yan et. al [16] use the Fuzzy C-Means (FCS) to cluster the observations of extended targets. Nevertheless, However, it only considered the linear tracking scenarios. Chen et. al [17] applied the cubature Kalman filter (CKF) into the ET-GM-PHD approach. It simply employ all of observations to compute the posterior intensity. When the number of targets grows large, the computational complexity increases greatly. The fuzzy adaptive resonance theory (ART) model has been presented for clustering the observations of extended targets in [12]. The estimating accuracy of this approach has greatly dependent on the selection of vigilance values. Wu et. al [18] presented an iterative random sample consensus to smooth observations in the sliding window. With the smoothed observations, the birth intensity can be adaptively initiated. However, this approach has been proposed under the point target assumption [19]. Inspired by [20], Peng et. al [21] presented an observation driven method to initiate the birth intensities of extended targets. Such a method assumes that there is only one birth born at each time step.

\section{Our work and contribution}

In this paper, we present the ET-GM-PHD approach with square-root cubature information filter (SCIF) to improve the tracking performance of the ET-GM-PHD approach. Here, 
we name our approach as the ET-SCI-GM-PHD approach. To be more specific, we, first, employ the cubature point to predict the mean and square-root of the covariance. In the light of the cubature information filter [22], the information forms of the predicted mean and covariance has been applied for achieving the posterior mean and covariance. Attribute to the significant tracking performance of the SCIF method, the ET-GM-PHD approach can be utilized to estimate states and number of multi extended targets with nonlinear dynamic models. Second, we present an observation driven method for initiating the birth intensity of our approach. Using such method, our approach can initiate the birth intensity adaptively. The main contributions are listed as follows,

1) We propose an improved ET-GM-PHD approach based on the SCIF and gating methods. First, we, under the ET-GM-PHD framework, use a group of GM components to represent the intensities of extended targets. Then, the cubature points have been used to predict the means and square-root of covariances of these GM components. Second, for saving the computational complexity, we compute the probability of each cell conditioned by the predicted observation. With the preset threshold, the candidate cells can be extracted from the current partition. On this basis, we tend to use the cell with largest value of the conditional probability as the cell corresponding to the predicted observation. Third, inspired by the cubature information filter, the information informs of the predicted mean and square-root of covariance has been adopted to formulate our SCIF method. Thus, using the extracted cell, the posterior mean and covariance can be achieved by the SCIF method. Finally, the posterior intensities of multi extended targets can be obtained for estimating states and number of extended targets.

2) We present an observation driven method for birth intensity initiating. Commonly, targets existed at current time step can not be considered as the birth targets. Thus, we use the estimated targets to approximate the birth intensity. Specifically, we, first, use the conditional probability to model the associations between targets and its corresponding cells. Then, we compute the probabilities of each partition conditioned by the estimated target set to achieve the most possible partition corresponding to the estimated target set. By removing cells associated with the estimated target set in the obtained partition, the intensity of the birth targets can be approximated. Thus, the birth intensity can be initiated adaptively.

The rest of this paper is organized as follows: We overview the PHD filter and GM-PHD implementation for extended targets in Section 2. Section 3 proposes our ET-GM-PHD approach. Simulation and comparison results are demonstrated in Section 4, and Section 5 concludes this paper.

\section{The PHD filter and GM-PHD for extended target tracking}

In this section, we introduce the random finite set 1 of the extended targets. Then, the formulations of the PHD filter for extended target tracking are represented in detail . At last, the GM-PHD implementation for extended targets is provided. The main notations are listed in Table 1.

\section{Observation Partitioning}

In multi extended target tracking scenarios, each state of targets may have more than one associated observations. In practice, the associations between observations are rather hard 
to be obtained. Therefore, observation partitioning becomes a great challenge for tracking extended targets. To illustrate such a challenge, let $\mathbf{Z}=\left\{\mathbf{z}_{1}, \mathbf{z}_{2}, \mathbf{z}_{3}\right\}$ be the observation set, where $\mathbf{z}_{i}$ denotes the $i$-th observation of $\mathbf{Z}$. The possible partitions of $\mathbf{Z}$ can be listed in the following [11]:

$\mathbf{C}_{1}: \mathbf{W}_{1}^{1}=\left\{\mathbf{z}_{1}, \mathbf{z}_{2}, \mathbf{z}_{3}\right\}$,

$\mathbf{C}_{2}: \mathbf{W}_{1}^{2}=\left\{\mathbf{z}_{1}\right\}, \mathbf{W}_{2}^{2}=\left\{\mathbf{z}_{2}, \mathbf{z}_{3}\right\}$,

$\mathbf{C}_{3}: \mathbf{W}_{1}^{3}=\left\{\mathbf{z}_{1}, \mathbf{z}_{2}\right\}, \mathbf{W}_{2}^{3}=\left\{\mathbf{z}_{3}\right\}$,

$\mathbf{C}_{4}: \mathbf{W}_{1}^{4}=\left\{\mathbf{z}_{1}, \mathbf{z}_{3}\right\}, \mathbf{W}_{2}^{4}=\left\{\mathbf{z}_{2}\right\}$,

$\mathbf{C}_{5}: \mathbf{W}_{1}^{5}=\left\{\mathbf{z}_{1}\right\}, \mathbf{W}_{2}^{5}=\left\{\mathbf{z}_{2}\right\}, \mathbf{W}_{3}^{5}=\left\{\mathbf{z}_{3}\right\}$,

where $\mathbf{W}_{j}^{i}$ is the $j$-th cell of partition $\mathbf{C}_{i}$.

Obviously, when the number of observations increases, the possible partitions grows even large. Under the PHD framework, computing the intensities of targets with these partitions may be rather expensive. In [23], a distance partition method has been proposed to solve such a problem. This method only utilizes the subset of partitions into the intensity computing, yields to better performance than the conventional Kmeans method. In this paper, we tend to adopt such a distance partition method.

\section{The PHD filter for the extended tracking}

Before introducing the basic idea of the PHD filter, we represent the nonlinear motion of the extended target in the following,

$$
\begin{array}{rc}
\text { Process model: } & \mathbf{x}_{k}=\varphi\left(\mathbf{x}_{k-1}\right)+\mathbf{v}_{k-1}, \\
\text { Observation model: } & \mathbf{z}_{k}=\phi\left(\mathbf{x}_{k}\right)+\mathbf{w}_{k}
\end{array}
$$

where $\varphi(\cdot)$ and $\phi(\cdot)$ denote the state transition and observation functions, respectively. $\mathbf{v}_{k}$ and $\mathbf{w}_{k}$ are the zero-mean Gaussian noises, and their covariances are denoted by $\mathbf{Q}_{k}$ and $\mathbf{R}_{k}$, respectively.

Let $D_{k-1}(\cdot)$ and $\mathbf{x}_{k-1}$ be the intensity and state of single extended target at time $k-1$. The predicted intensity can be described by (3).

$$
D_{k \mid k-1}\left(\mathbf{x}_{k} \mid \mathbf{Z}_{1: k-1}\right)=\underbrace{\int_{\mathfrak{X}} p_{s}\left(\mathbf{x}_{k}\right) \varphi_{k \mid k-1}\left(\mathbf{x}_{k} \mid \mathbf{x}\right) D_{k-1 \mid k-1}\left(\mathbf{x} \mid \mathbf{Z}_{1: k-1}\right) d \mathbf{x}}_{\text {Survival intensity }}+\underbrace{\gamma_{k}\left(\mathbf{x}_{k}\right)}_{\text {Birth intensity }},
$$

where $p_{s}(\cdot)$ is the survival probability of single target, $f(\cdot)$ denotes the transition density, $\mathbf{Z}_{1: k-1}$ is the observation sets from time 1 to $k-1$, and $\gamma(\cdot)$ represent the intensity of birth target.

Then, the predicted $D_{k \mid k-1}\left(\mathbf{x}_{k} \mid \mathbf{Z}_{1: k-1}\right)$ can be updated by

$$
D_{k}\left(\mathbf{x}_{k}\right)=L_{z}\left(\mathbf{x}_{k}\right) D_{k \mid k-1}\left(\mathbf{x}_{k} \mid \mathbf{Z}_{1: k-1}\right)
$$

where $L_{z}(\cdot)$ is a pseudolikelihood function, represented by (5).

$$
L_{z}\left(\mathbf{x}_{k}\right)=1-\left(1-e^{\beta\left(\mathbf{x}_{k}\right)}\right) p_{D}\left(x_{k}\right)+e^{\beta\left(\mathbf{x}_{k}\right)} p_{D}\left(\mathbf{x}_{k}\right) \sum_{\mathbf{y} \angle \mathbf{Z}_{k}} \omega_{\mathbf{y}} \sum_{W \angle \mathbf{y}} \frac{\beta\left(\mathbf{x}_{k}\right)^{\left|\beta\left(\mathbf{x}_{k}\right)\right|}}{d_{W}} \cdot \prod_{\mathbf{z} \in \mathbf{W}} \frac{\phi_{W}\left(\mathbf{x}_{k}\right)}{\lambda_{k} c_{k}(\mathbf{z})} .
$$


As for (5), $\beta(\cdot)$ denotes the expected number of measurements generated by single extended target, $p_{D}(\cdot)$ is the detection probability of single target. $\phi_{W}\left(\mathbf{x}_{k}\right)$ refers to the likelihood of single target. $\mathbf{y} \angle \mathbf{Z}_{k}$ means that $\mathbf{Z}_{k}$ is partitioned into nonempty cells $W$ by $\mathbf{y}$. $\omega_{\mathbf{y}}$ and $d_{W}$ are nonnegative coefficients, expressed by

$$
\omega_{\mathbf{y}}=\frac{\prod_{\mathbf{W} \in \mathbf{y}} d_{\mathbf{W}}}{\sum_{\mathbf{y} \angle \mathbf{z}_{k}} \prod_{\mathbf{W}} d_{\mathbf{W}}}
$$

where $d_{\mathbf{W}}$ is expressed by (7).

$$
d_{\mathbf{W}}=\delta_{|\mathbf{W}|, 1}+\int D_{k \mid k-1}\left(\mathbf{x} \mid \mathbf{Z}_{1: k-1}\right) e^{\beta(\mathbf{x})} \beta(\mathbf{x})^{|\beta(\mathbf{x})|} p_{D}(\mathbf{x}) \prod_{\mathbf{z} \in \mathbf{W}} \frac{\phi\left(\mathbf{x}_{k}\right)}{\lambda_{k} c_{k}(\mathbf{z})} d \mathbf{x},
$$

where $\delta_{i, j}$ is the Kronecker delta.

Equations (3) and (4) describe a PHD recursion in extended target cases. Similar to the standard target cases, there are no closed-forms for (3) and (4).

\section{GM-PHD for extended target tracking}

In this section, we overview the basic idea of the ET-GM-PHD approach. It can be considered as the GM implementation of the PHD approach in Section. As for this approach, the predicted and updated intensities are constructed by the GM components, respectively. Using these GM components, (3) and (4) can be converted into the summation of GM components. Thus, these equations can be used to estimate states of targets. The details of the ET-GM-PHD approach are provided in the following.

According to [23], the intensity $D_{k-1}(\mathbf{x})$ are constructed by a group of GM components, represented by

$$
\mathbf{D}_{k-1}=\sum_{j=1}^{J_{k-1}} w_{k-1}^{j} N\left(\mathbf{x} ; \mathbf{m}_{k-1}^{j}, \mathbf{P}_{k-1}^{j}\right)
$$

where, $\mathbf{m}_{k-1}^{j}$ and $\mathbf{P}_{k-1}^{j}$ denote the mean and covariance of the $j$-th component. $w_{k-1}^{j}$ represent the weight of the $j$-th component, and $J_{k-1}$ is the number of GM component at time step $k-1$.

Using (8), the predicted intensity of (3) at time $k$ can be computed by

$$
D_{k \mid k-1}(\mathbf{x})=v_{s, k \mid k-1}(\mathbf{x})+\gamma_{k}(\mathbf{x}),
$$

where $v_{s, k \mid k-1}(\mathbf{x})$ and $\gamma_{k}(\mathbf{x})$ denote the intensities of survival and birth components, respectively. $v_{s, k \mid k-1}(\mathbf{x})$ can be formulated by

$$
v_{s, k \mid k-1}(\mathbf{x})=p_{s, k} \sum_{j=1}^{J_{k-1}} w_{k-1}^{j} N\left(\mathbf{x} ; \mathbf{m}_{k \mid k-1}^{j}, \mathbf{P}_{k \mid k-1}^{j}\right),
$$


$\mathbf{m}_{k \mid k-1}^{j}$, and $\mathbf{P}_{k \mid k-1}^{j}$ represent the predicted state mean and covariance of the $j-$ th component, respectively. Moreover, we construct $\gamma_{k}(\mathbf{x})$ by

$$
\gamma_{k}(\mathbf{x})=\sum_{i=1}^{J_{r, k}} w_{r, k}^{i} N\left(\mathbf{x} ; \mathbf{m}_{r, k}^{i}, \mathbf{P}_{r, k}^{i}\right)
$$

where $J_{r, k}$ is the number of birth Gaussian mixture components, $w_{r, k}^{i}$ is the weight of the $i$-th component. $\mathbf{m}_{r, k}^{i}$ and $\mathbf{P}_{r, k}^{i}$ denote the mean and covariance, respectively.

Then, the predicted intensity $D_{k \mid k-1}(\mathbf{x})$ can be updated by

$$
D_{k}(\mathbf{x})=D_{k}^{N D}(\mathbf{x})+D_{k}^{D}(\mathbf{x})
$$

where $D_{k}^{N D}(\mathbf{x})$ denotes the no detection case,

$$
\begin{aligned}
D_{k}^{N D}(\mathbf{x}) & =\sum_{j=1}^{J_{k \mid k-1}} w_{k \mid k}^{j} N\left(x ; \mathbf{m}_{k}^{j}, \mathbf{P}_{k}^{j}\right) \\
w_{k}^{j} & =\left(1-\left(1-e^{-\beta(\mathbf{x})}\right)\right) p_{D}(\mathbf{x}) w_{k \mid k-1}^{j} \\
\mathbf{m}_{k \mid k}^{j} & =\mathbf{m}_{k \mid k-1}^{j}, \mathbf{P}_{k \mid k}^{j}=\mathbf{P}_{k \mid k-1}^{j},
\end{aligned}
$$

and $D_{k \mid k}^{D}(\mathbf{x})$ is the detected target case,

$$
\begin{aligned}
D_{k}^{D}(\mathbf{x}) & =\sum_{j=1}^{J_{k \mid k-1}} w_{k \mid k}^{j} N\left(x ; \mathbf{m}_{k}^{j}, \mathbf{P}_{k}^{j}\right), \\
w_{k}^{j} & =\omega_{y} \frac{\Gamma^{j} p_{D}(\mathbf{x})}{d_{W}} \Phi_{W}^{j} w_{k \mid k-1}^{j}, \\
\Gamma^{j} & =e^{-\beta(\mathbf{x})}-\beta(\mathbf{x})^{|\beta(\mathbf{x})|} \\
\Phi_{W}^{j} & =\phi_{W}(\mathbf{x}) \prod_{\mathbf{z} \in \mathbf{W}} \frac{1}{\lambda_{k} c_{k}(\mathbf{z})}
\end{aligned}
$$

where the coefficient $\phi_{W}(\mathbf{x})$ can be computed by

$$
\phi_{W}(\mathbf{x})=N\left(\mathbf{z}_{W} ; \mathbf{z z}_{k \mid k-1}, \mathbf{P}_{W, k \mid k-1}^{z z}\right)
$$

where $\mathbf{z z}_{k \mid k-1}$ and $\mathbf{P}_{W, k \mid k-1}^{z z}$ are the predicted observation and its corresponding covariance, respectively.

$$
\mathbf{z}_{W}=\bigoplus_{\mathbf{z}_{k} \in \mathbf{W}} \mathbf{z}_{k}
$$

Here, $\bigoplus$ denotes the vertical vectorial concatenation.

Equations (9)-(19) are the main formulations of the ET-GM-PHD approach. Implementing these equations iteratively, the posterior intensities of the extended targets can be conveniently obtained. Actually, for most of the ET-GM-PHD approaches, $\phi(\cdot)$ and $\varphi(\cdot)$ of (1) and (2) are assumed as linear functions. Thus, $\mathbf{z z}_{k \mid k-1}$ of (20) can be obtained by

$$
\mathbf{z z}_{k \mid k-1}=\mathbf{H}_{W} \mathbf{m}_{k \mid k-1}
$$


where

$$
\begin{aligned}
& \mathbf{H}_{W}=\operatorname{repmat}\left(\mathbf{H}_{w},|\mathbf{W}|, 1\right), \\
& \operatorname{repmat}\left(\mathbf{H}_{w},|\mathbf{W}|, 1\right)=[\underbrace{\mathbf{H}_{k}^{T}, \ldots, \mathbf{H}_{k}^{T}}_{|\mathbf{W}| \text { times }}]
\end{aligned}
$$

where $\mathbf{H}_{k}$ is the coefficient matrix. Using (22), $\mathbf{m}_{k \mid k}^{j}$ and $\mathbf{P}_{k \mid k}^{j}$ can be achieved by the Kalman filter [23].

Equation (22) denotes the estimation of the predicted observations in linear scenarios. When targets are with the nonlinear motions, it cannot be adopted to estimate predicted observation.

\section{Methods}

In this section, we propose the ET-SCI-GM-PHD approach for tracking extended targets in nonlinear scenarios. Specifically, we, first, present the SCIF based ET-GM-PHD approach for estimating the number and states of the multi extended targets. Then, an observation driven initiation method has been presented for initiating the birth intensity. Thus, the birth intensity of our approach can be adaptively initiated.

\section{SCIF Based ET-GM-PHD Approach for extended target tracking}

Recall that $v_{s, k \mid k-1}(\mathbf{x})$ and $D_{k \mid k}^{D}(\mathbf{x})$ in (10) and (16) consist of GM components, these GM components can be obtained by computing the means and covariances of the corresponding components. Similar to the point target tracking, we tend to use the SCKF method to estimate these means and covariances. Although the SCKF method has high estimating accuracy in nonlinear target tracking, it has been proposed under the point target assumption. Thus, the SCKF method cannot been directly used in extended target tracking scenarios. For applying the SCK method into extended target scenarios, we, first, directly use the SCKF method to obtain the predicted means and square-root of covariances of GM components in (10). By updating these information forms, the posterior mean and covariance can be achieved. Then, inspired by the CIF method, we estimate the state and square-root of the covariance by propagating the information forms of the predicted mean and square-root of covariance. Meanwhile, for reducing the influence of clutters, we utilize a gating method to construct the cell of the current partition, which can be used in the updating stage of our SCIF method.

Before introducing our approach, we use $\mathbf{m}_{k-1}$ and $\mathbf{P}_{k-1}$ to replace $\mathbf{m}_{k-1}^{j}$ and $\mathbf{P}_{k-1}^{j}$ of the $j$-th component of (8). The SCKF based ET-GM-PHD approach consists of the following steps,

\section{Calculate the predicted GM components}

In (9), the predicted density $v_{s, k \mid k-1}(\mathbf{x})$ is constructed by GM components. These components can be calculated by estimating the corresponding means and covariances, which can be illustrated in the following,

Let $\mathbf{S}_{k-1}$ be the square-root factor of $\mathbf{P}_{k-1}, \mathbf{S}_{k-1}$ can be obtained by the Cholesky decomposition of $\mathbf{P}_{k-1}$. Obviously, we have

$$
\mathbf{P}_{k-1}=\mathbf{S}_{k-1} \mathbf{S}_{k-1}^{T}
$$


where $(\cdot)^{T}$ represents the transpose operation.

According to (25), the predicted covariance $\mathbf{P}_{k \mid k-1}$ can be acquired by calculating $\mathbf{S}_{k \mid k-1}$. As for the SCKF method, $\mathbf{m}_{k \mid k-1}$ and $\mathbf{S}_{k \mid k-1}$ are estimated by a group of the cubature points [24]. In this paper, we use the set $\left\{\boldsymbol{\alpha}_{j}, \rho_{j}\right\}$ to represent the cubature point set, where

$$
\left\{\begin{array}{rl}
\boldsymbol{\alpha}_{j} & =\sqrt{d}[\mathbf{1}]_{j} \\
\rho_{j} & =\frac{1}{2 d}
\end{array}, j=1,2, \ldots, 2 d\right.
$$

$d$ denotes the dimension of $\mathbf{m}_{k-1}$, and $[\mathbf{1}]_{j}$ is the $j$-th vector of the set

$$
\left\{\left[\begin{array}{l}
1 \\
0 \\
\vdots \\
0
\end{array}\right], \cdots,\left[\begin{array}{l}
0 \\
\vdots \\
0 \\
1
\end{array}\right],\left[\begin{array}{l}
-1 \\
0 \\
\vdots \\
0
\end{array}\right],\left[\begin{array}{l}
0 \\
\vdots \\
0 \\
-1
\end{array}\right]\right\}
$$

With the cubature point set $\left\{\boldsymbol{\alpha}_{j}, \rho_{j}\right\}$, the predicted mean $\mathbf{m}_{k \mid k-1}$ and square-root factor $\mathbf{S}_{k \mid k-1}$ can be computed as follows:

1) Estimate the cubature points $(j=1, \ldots, 2 d)$

$$
\chi_{k-1, j}=\mathbf{S}_{k-1} \boldsymbol{\alpha}_{j}+\mathbf{m}_{k-1}
$$

2) Calculate the propagated cubature points $(j=1, \ldots, 2 d)$

$$
\chi_{k-1, j}^{*}=\varphi\left(\chi_{k-1, j}\right)
$$

3) Predict the mean of GM component

$$
\mathbf{m}_{k \mid k-1}=\sum_{j=1}^{2 n} \rho_{j} \chi_{k-1, j}^{*}
$$

4) Evaluate the predicted square-root factor

$$
\mathbf{S}_{k \mid k-1}=\operatorname{Tria}\left(\left[\mathbf{C}_{k \mid k-1, j}, \mathbf{S}_{Q, k-1}\right]\right)
$$

where

$$
\begin{aligned}
& \mathbf{C}_{k \mid k-1, j}=\frac{1}{\sqrt{2 d}} \quad\left[\chi_{k-1,1}^{*}-\mathbf{m}_{k \mid k-1} \quad \ldots\right. \\
& \left.\chi_{k-1,2 n}^{*}-\mathbf{m}_{k \mid k-1}\right],
\end{aligned}
$$

Tria $(\cdot)$ denotes the QR decomposition, and $\mathbf{S}_{Q, k-1}$ is the square root factor of $\mathbf{Q}_{k-1}$.

Substituting $\mathbf{S}_{k \mid k-1}$ of (30) into (25), we can achieve the predicted covariance $\mathbf{P}_{k \mid k-1}$. Iterating (27) to (30), the GM components of (10) can be achieved. 
The conventional ET-GM-PHD uses all of cells in current partition to calculate the updated mean $\mathbf{m}_{k}$ and square-root of covariance $\mathbf{S}_{k \mid k-1}$. When the number of cells grows large, it may cost great computational complexity. To solve such a problem, we tend to extract the most possible cell from the current partition.

In extended target tracking scenarios, each partition consists of several cells. Directly using these cells may cost great the computational complexity. To avoid such a problem, we only use part of cells. Here, we name these cells as the possible cells. In order to obtain the possible cells, we define the probability between the current cell and predicted observation by $p\left(\mathbf{Z}_{g, k} \mid \mathbf{z}_{k \mid k-1}\right)$. Here, $\mathbf{Z}_{g, k}$ is the $g$-th cell of the current partition. $\mathbf{z}_{k \mid k-1}$ is the predicted observation, which can be computed by

$$
\begin{aligned}
& \mathbf{z}_{k \mid k-1}=\sum_{j=1}^{2 d} \rho_{j} \boldsymbol{\chi}_{k \mid k-1, j}^{*}, \\
& \chi_{k \mid k-1, j}^{*}=\varphi\left(\boldsymbol{\chi}_{k \mid k-1, j}\right), \\
& \chi_{k \mid k-1, j}=\mathbf{S}_{k \mid k-1} \boldsymbol{\alpha}_{j}+\boldsymbol{m}_{k \mid k-1} .
\end{aligned}
$$

Recall that observations in the same cell represent observations generated by the same target, these observations are independent identically distributed. Thus, we have

$$
\begin{aligned}
p\left(\mathbf{Z}_{g, k} \mid \mathbf{z}_{k \mid k-1}\right) & =p\left(\mathbf{z}_{1, k}^{g}, \mathbf{z}_{2, k}^{g}, \ldots, \mathbf{z}_{|\mathbf{W}|, k}^{g} \mid \mathbf{z}_{k \mid k-1}\right), \\
& =\prod_{j=1}^{|\mathbf{W}|} p\left(\mathbf{z}_{j, k}^{g} \mid \mathbf{z}_{k \mid k-1}\right)
\end{aligned}
$$

where $\mathbf{z}_{j, k}^{g}$ is the $j$-th observation of $\mathbf{Z}_{g, k}$, and $|\mathbf{W}|$ is the number of observations in $\mathbf{Z}_{g, k}$. According to (2), $p\left(\mathbf{z}_{j, k} \mid \mathbf{z}_{k \mid k-1}\right)$ can be represented by

$$
p\left(\mathbf{z}_{j, k}^{g} \mid \mathbf{x}_{k}\right)=N\left(\mathbf{z}_{j, k} ; \mathbf{z}_{k \mid k-1}, \mathbf{R}_{k}\right)
$$

Substituting (36) into (35), the probability $p\left(\mathbf{Z}_{g, k} \mid \mathbf{z}_{k \mid k-1}\right)$ can be rewritten by

$$
p\left(\mathbf{Z}_{g, k} \mid \mathbf{z}_{k \mid k-1}\right)=\prod_{j=1}^{|\mathbf{W}|} N\left(\mathbf{z}_{j, k}^{g} ; \mathbf{z}_{k \mid k-1}, \mathbf{R}_{k}\right) .
$$

Obviously, when $\mathbf{Z}_{g, k}$ is associated with $\mathbf{z}_{k \mid k-1}$, the probability $p\left(\mathbf{Z}_{g, k} \mid \mathbf{z}_{k \mid k-1}\right)$ in (37) may have large value. Thus, the candidate cell set can be achieved by

$$
\tilde{\mathbf{C}}_{k}=\left\{\mathbf{Z}_{g, k} \mid p\left(\mathbf{Z}_{g, k} \mid \mathbf{z}_{k \mid k-1}\right)<T_{h}\right\}
$$

where $T_{h}$ is the preset threshold.

Therefore, the most possible cell associated with $\mathbf{z}_{k \mid k-1}$ can be extracted by

$$
\mathbf{W}_{C}=\arg \max _{\mathbf{Z}_{g, k}} p\left(\mathbf{Z}_{g, k} \mid \mathbf{z}_{k \mid k-1}\right)
$$


where $\mathbf{Z}_{g, k} \in \tilde{\mathbf{C}}_{k}$.

\section{Evaluating the updated mean and covariance}

Under the CIF framework, the information forms of the predicted mean and covariance $\mathbf{P}_{k \mid k-1}$ can be represented by

$$
\begin{aligned}
\mathbf{y}_{k \mid k-1} & =\mathbf{Y}_{k \mid k-1} \mathbf{m}_{k \mid k-1} \\
\mathbf{Y}_{k \mid k-1} & =\left(\mathbf{P}_{k \mid k-1}\right)^{-1},
\end{aligned}
$$

where $\mathbf{P}_{k \mid k-1}=\mathbf{S}_{k \mid k-1} \mathbf{S}_{k \mid k-1}^{T}$.

Using (40)-(41), the state contribution and its corresponding information matrix can be computed by

$$
\mathbf{i}_{k, j}=\mathbf{Y}_{\mathbf{k} \mid \mathbf{k}-\mathbf{1}} \mathbf{P}_{k \mid k-1}^{m z} \mathbf{R}_{k}^{-1}\left(\boldsymbol{\mu}_{j}+\left(\mathbf{Y}_{\mathbf{k} \mid \mathbf{k}-\mathbf{1}} \mathbf{P}_{k \mid k-1}^{m z}\right)^{T} \mathbf{m}_{k \mid k-1}\right)
$$

and

$$
\mathbf{I}_{k, j}=\mathbf{Y}_{\mathbf{k} \mid \mathbf{k}-\mathbf{1}} \mathbf{P}_{k \mid k-1}^{m z} \mathbf{R}_{k, j}^{-1}\left(\mathbf{Y}_{\mathbf{k} \mid \mathbf{k}-\mathbf{1}} \mathbf{P}_{k \mid k-1}^{m z}\right)^{T}
$$

where $\mathbf{R}_{k, j}$ is the $j$-th observation, $\boldsymbol{\mu}_{j}$ represents the innovation of the $j$-th observation $\mathbf{z}_{j}$,

$$
\boldsymbol{\mu}_{j}=\mathbf{z}_{j}-\mathbf{z}_{k \mid k-1}
$$

Here, $\mathbf{z}_{j}$ is the $j$-th component of the observation set $\tilde{\mathbf{Z}}_{k}$, and $\tilde{\mathbf{Z}}_{k}$ represents the observation set of the current target. We also assume that $\mathbf{z}_{j}$ is a two-dimension vector, and $\boldsymbol{\mu}_{j}$ follows a two-dimension Gaussian distribution. That is to say, these observations have the same covariances of observation noises.

Thus, we can obtain the information state vector $\mathbf{y}_{k}$ and matrix $\mathbf{Y}_{k}$ by

$$
\begin{aligned}
\mathbf{y}_{k} & =\mathbf{y}_{k \mid k-1}+\sum_{j=1}^{N_{l}} \mathbf{i}_{k, j} \\
\mathbf{Y}_{k} & =\mathbf{Y}_{k \mid k-1}+\sum_{j=1}^{N_{l}} \mathbf{I}_{k, j}, \\
& =\mathbf{Y}_{k \mid k-1}+N_{l} \cdot \mathbf{I}_{k} .
\end{aligned}
$$

From (45)-(46), we can observe that the CIF method method uses the information forms of the state and covariance to calculate the posterior state and covariance. When the covariance matrix is indefinite, the CIF method can not be implemented correctly. For achieving the positive definite covariance matrix, we, update the covariance by propagating the information form of its square-root factor. With the propagated information form of its square root factor, the square-root factor of the covariance can be computed, which can be used to calculate the posterior covariance.

Recall that $\mathbf{S}_{k \mid k-1}$ is the square root factor of $\mathbf{P}_{k \mid k-1}$, we denote the information form of $\mathbf{S}_{k \mid k-1}$ as

$$
\mathbf{Y}_{s, k \mid k-1}=\mathbf{S}_{k \mid k-1}^{-1}
$$


Obviously, $\mathbf{Y}_{k \mid k-1}$ can be represented by

$$
\begin{aligned}
\mathbf{Y}_{k \mid k-1} & =\left(\mathbf{Y}_{k \mid k-1}\right)^{-1} \\
& =\left(\mathbf{S}_{k \mid k-1} \mathbf{S}_{k \mid k-1}^{T}\right)^{-1} \\
& =\mathbf{Y}_{s, k \mid k-1}^{T} \mathbf{Y}_{s, k \mid k-1}
\end{aligned}
$$

Thus, $\mathbf{Y}_{k \mid k-1}$ can be obtained by calculating $\mathbf{Y}_{s, k \mid k-1}$.

Let $\mathbf{S}_{R, k}$ be the square root of $\mathbf{R}_{k}$, its corresponding information form is denoted by $\mathbf{Y}_{s r, k}$. With the obtained $\mathbf{Y}_{k \mid k-1}$ and $\mathbf{Y}_{s r, k}$, (45) and (46) can be rewritten by

$$
\begin{aligned}
\mathbf{I}_{k} & =\mathbf{M}_{k} \mathbf{Y}_{s r, k}^{T} \mathbf{Y}_{s r, k} \mathbf{M}_{k}^{T} \\
& =\mathbf{Y}_{I, k} \mathbf{Y}_{I, k}^{T}, \\
\mathbf{i}_{k, j} & =\mathbf{Y}_{I, k} \mathbf{Y}_{s r, k}\left(\boldsymbol{\mu}_{j}+\mathbf{M}_{k}^{T} \mathbf{m}_{k \mid k-1}\right),
\end{aligned}
$$

where

$$
\mathbf{M}_{k}=\mathbf{Y}_{s, k \mid k-1}^{T} \mathbf{Y}_{s, k \mid k-1} \mathbf{P}_{k \mid k-1}^{m z}
$$

and

$$
\mathbf{Y}_{I, k}=\mathbf{M}_{k} \mathbf{Y}_{s r, k}^{T}
$$

Substitute (49) into (46), $\mathbf{Y}_{k}$ can be represented by

$$
\begin{aligned}
\mathbf{Y}_{k}= & \mathbf{Y}_{k \mid k-1}+N_{l} \cdot \mathbf{I}_{k} \\
= & \mathbf{Y}_{s, k \mid k-1}^{T} \mathbf{Y}_{s, k \mid k-1}+N_{l} \cdot \mathbf{Y}_{I, k} \mathbf{Y}_{I, k}^{T} \\
= & {\left[\mathbf{Y}_{s, k \mid k-1}^{T}, \sqrt{N_{l}} \cdot \mathbf{Y}_{I, k}\right] } \\
& \times\left[\mathbf{Y}_{s, k \mid k-1}^{T}, \sqrt{N_{l}} \cdot \mathbf{Y}_{I, k}\right]^{T}
\end{aligned}
$$

Hence, using matrix triangularization of (54), the information form of $\mathbf{S}_{k}$ can be formulated by

$$
\mathbf{Y}_{s, k}=\left(\operatorname{Tria}\left(\left[\mathbf{Y}_{s, k \mid k-1}^{T}, \sqrt{N_{l}} \cdot \mathbf{Y}_{I, k}\right)\right)^{T}\right.
$$

In addition, when $\mathbf{Z}_{g, k}$ is empty, we let

$$
\mathbf{Y}_{s, k}=\mathbf{Y}_{s, k \mid k-1}
$$

and

$$
\mathbf{m}_{k}=\mathbf{m}_{k \mid k-1} .
$$

According to (47) and (55), $\mathbf{S}_{k}$ can be achieved. With the calculated $\mathbf{S}_{k}, \mathbf{P}_{k}$ can be obtained. Then, we can compute the density $D_{k}^{D}(\mathbf{x})$ of (16) by computing each component. Substituting the obtained $D_{k}^{D}(\mathbf{x})$ and $D_{k}^{N D}(\mathbf{x})$ into (12), we finally achieve the density $D_{k}(\mathbf{x})$. 


\section{An observation-driven method for birth initiation}

As for the conventional ET-GM-PHD approaches, the birth intensities are commonly assumed as a prior. In practice, these intensities are always unknown. For initiating the birth intensities, we, in this paper, presented a birth intensity initiation method using the estimated targets. To be more specific, we, first, compute the probability of each pair of the target and cell. Using the likelihood, we can obtain the probability of each partition conditioned by the estimated target set. Then, the probability with largest value can be consider as the most possible partition. On this basis, we remove the cells associated with the estimated targets. The left cells are utilized to initiate the birth intensity.

Recall the observation model of (2), the conditional probability between $\mathbf{x}_{k}$ and $\mathbf{z}_{k}$ can be represented by

$$
p\left(\mathbf{z}_{k} \mid \mathbf{x}_{k}\right)=N\left(\mathbf{z}_{k} \mid \phi\left(\mathbf{x}_{k}\right), \mathbf{R}_{k}\right)
$$

Then, the conditional probability between $\mathbf{x}_{k}$ between cell $\mathbf{Z}_{g, k}$ can be denoted by

$$
p\left(\mathbf{Z}_{g, k} \mid \mathbf{x}_{k}\right)=\prod_{j=1}^{|\mathbf{W}|} N\left(\mathbf{z}_{j, k}^{g} ; \phi\left(\mathbf{x}_{k}\right), \mathbf{R}_{k}\right)
$$

From (59), we can observe that when $\mathbf{Z}_{g, k}$ is generated by $\mathbf{x}_{k}$, (59) may have large value. Let $\tilde{\mathbf{x}}_{k, i}$ be the state of the $i$-th target in estimated set $\tilde{\mathbf{X}}_{k}$. Therefore, the cell generated by $\tilde{\mathbf{x}}_{k, i}$ can be represented by

$$
\begin{array}{r}
\tilde{\mathbf{W}}_{k, i}=\quad \arg \max _{\mathbf{Z}_{g, k}} p\left(\mathbf{Z}_{g, k} \mid \tilde{\mathbf{x}}_{k, i}\right) \\
\text { s.t. } \quad p\left(\mathbf{Z}_{g, k} \mid \tilde{\mathbf{x}}_{k, i}\right)>T_{h} .
\end{array}
$$

Here, $T_{h}$ is the preset threshold, which is used to decrease the influence of $\mathbf{Z}_{g, k}$ with small value of $p\left(\mathbf{Z}_{g, k} \mid \tilde{\mathbf{x}}_{k, i}\right)$.

Using (60), the probability of the partition $\mathbf{C}_{g, k}$ conditional by the estimated target set $\tilde{\mathbf{X}}_{k}$ can be defined by

$$
\begin{aligned}
p\left(\mathbf{C}_{g, k} \mid \tilde{\mathbf{X}}_{k}\right) & =p\left(\tilde{\mathbf{W}}_{k, 1}, \ldots, \tilde{\mathbf{W}}_{k, N_{x}} \mid \tilde{\mathbf{x}}_{k, 1}, \ldots, \tilde{\mathbf{x}}_{k, N_{X}}\right) \\
& =\frac{\prod_{i=1}^{N_{X}} p\left(\tilde{\mathbf{W}}_{k, i}, \tilde{\mathbf{x}}_{k, i}\right)}{\prod_{i=1}^{N_{X}} p\left(\tilde{\mathbf{x}}_{k, i}\right)} \\
& =\prod_{i=1}^{N_{x}} p\left(\tilde{\mathbf{W}}_{k, i} \mid \tilde{\mathbf{x}}_{k, i}\right)
\end{aligned}
$$

where $N_{X}=\left|\tilde{\mathbf{X}}_{k}\right|$ denotes the number of estimated targets.

Similar to (60), the most possible partition associated with $\tilde{\mathbf{X}}_{k}$ may have the largest probability (calculated by (61)). Hence, we have

$$
\tilde{\mathbf{C}}_{k}=\arg \max _{\mathbf{C}_{g, k}} p\left(\mathbf{C}_{g, k} \mid \tilde{\mathbf{X}}_{k}\right)
$$

where $g=1,2, \ldots, N_{C}$, and $N_{C}$ is the number of possible partitions. 
Since the candidate partition can be obtained by (62), observations generated by the birth targets can be extracted by removing cells associated with the estimated targets. Therefore, the cell set associated with birth targets can be represented by

$$
\tilde{\mathbf{C}}_{k}^{b}=\tilde{\mathbf{C}}_{k} \backslash\left\{\tilde{\mathbf{W}}_{k, 1}, \ldots, \tilde{\mathbf{W}}_{k, N_{x}}\right\}
$$

In order to initiate the birth intensity, we combine the cell of the birth target into one observation. Let $\tilde{\mathbf{W}}_{j, k}^{b}$ be the $j$-th cell of $\tilde{\mathbf{C}}_{k}^{b}$, for initiating the birth intensity, we tend to combine observations of $\tilde{\mathbf{W}}_{j, k}^{b}$ by

$$
\tilde{\mathbf{z}}_{j, k}^{b}=\frac{1}{\left|\tilde{\mathbf{W}}_{j, k}^{b}\right|} \sum_{i=1}^{\left|\tilde{\mathbf{W}}_{j, k}^{b}\right|} \mathbf{z}_{i, j, k}^{b} .
$$

With the combined observation $\tilde{\mathbf{z}}_{j, k}^{b}$, we use the unbiased observation model in [25] to project $\tilde{\mathbf{z}}_{j, k}^{b}$ into the state space. For simplicity, we use $\tilde{\mathbf{z}}$ to instead of $\tilde{\mathbf{z}}_{j, k}^{b}$. Let $\theta_{k}$ and $r_{k}$ be the bearing angle and range of $\tilde{\mathbf{z}}$, the projected state of $\tilde{\mathbf{z}}$ can be represented by $\tilde{\mathbf{m}}_{k}^{b}=\left[s_{k}^{x}, 0, s_{k}^{y}, 0,0\right]^{T}$, where

$$
\begin{aligned}
s_{k}^{x} & =\beta_{\theta}^{-1} r_{k} \cos \theta_{k}, \\
s_{k}^{y} & =\beta_{\theta}^{-1} r_{k} \sin \theta_{k} .
\end{aligned}
$$

In (65) and (66), $\beta_{\theta}$ is the biased comparison factor. Here, we set $\beta_{\theta}=\sigma_{\theta}$, and $\sigma_{\theta}$ is the error of the bearing angle.

In addition, according to [25], the covariance of $\tilde{\mathbf{m}}_{k}^{b}$ can be expressed by

$$
\mathbf{P}_{k}^{b}=\left[\begin{array}{ccccc}
\sigma_{x x} & 0 & \sigma_{x y} & 0 & 0 \\
0 & \sigma_{v} & 0 & 0 & 0 \\
\sigma_{y y} & 0 & \sigma_{x y} & 0 & 0 \\
0 & 0 & 0 & \sigma_{v}^{2} & 0 \\
0 & 0 & 0 & 0 & \sigma_{\theta}^{2}
\end{array}\right]
$$

where, $\sigma_{v}$ is the error of the velocity,

$$
\left\{\begin{aligned}
\sigma_{x x}= & \left(\beta_{\theta}^{-2}-2\right)\left(\tilde{r}_{k, i}\right)^{2} \cos ^{2}\left(\theta_{k, i}\right)+0.5\left(\left(\tilde{r}_{k, i}\right)^{2}\right. \\
& \left.+\sigma_{r}^{2}\right)\left(1+\beta_{\theta}^{4} \cos \left(2 \theta_{k, i}\right)\right) \\
\sigma_{x y}= & \left(\beta_{\theta}^{-2}-2\right)\left(\tilde{r}_{k, i}\right)^{2} \cos \left(\theta_{k, i}\right) \sin \left(\theta_{k, i}\right)+0.5\left(\left(\tilde{r}_{k, i}\right)^{2}\right. \\
& \left.+\sigma_{r}^{2}\right)\left(1+\beta_{\theta}^{4} \cos \left(2 \theta_{k, i}\right)\right) \\
\sigma_{y y}= & \left(\beta_{\theta}^{-2}-2\right)\left(\tilde{r}_{k, i}\right)^{2} \sin ^{2}\left(\theta_{k, i}\right)+0.5\left(\left(\tilde{r}_{k, i}\right)^{2}\right. \\
& \left.+\sigma_{r}^{2}\right)\left(1-\beta_{\theta}^{4} \cos \left(2 \theta_{k, i}\right)\right)
\end{aligned}\right.
$$

Using (65)-(67), the Gaussian mixture components of birth targets can be constructed by $\left\{\mathbf{m}_{k, i}^{b}, \mathbf{P}_{k, i}^{b}\right\}_{i=1}^{N_{k}, b}$, where $N_{k}^{b}$ is the cell number of $\tilde{\mathbf{C}}_{k}^{b}$. Then, these components are used to initiate the birth intensity $\gamma_{k}(\mathbf{x})$ of (9). We summarize the proposed ET-SCI-GM-PHD approach in Table 2 . 


\section{Results and Discussion}

In this section, we validate the tracking performance of our ET-SCI-GM-PHD approach. We construct a nonlinear scenario composed of five target. Under such a scenario, we use the optimal subpattern assignment (OSPA) metric [26] and Root Mean Square Error (RMSE) to compare the tracking performance of the ET-GM-PHD [23], CK-EPHD [17] and our ET-SCI-GM-PHD approaches. We compare the simulation results of the three approaches under certain number of clutters, various clutter numbers, and detected probabilities.

\section{Simulation Scenarios}

In this section, we follow the way of [27] to construct the nonlinear scenarios. Let $\mathbf{x}_{k}=$ $\left[s_{k}^{x}, v_{k}^{x}, s_{k}^{x}, v_{k}^{y}, \alpha_{k}\right]$, where $\left(v_{k}^{x}, v_{k}^{y}\right)$ is the velocity, $\left(s_{k}^{x}, s_{k}^{y}\right)$ is the position, and $\alpha_{k}$ is the turn rate. With these definitions, we represent the nonlinear dynamic model by

$$
\begin{gathered}
\mathbf{x}_{k}= \\
{\left[\begin{array}{ccccc}
1 & \frac{\sin \left(\alpha_{k-1} T\right)}{\alpha_{k-1}} & 0 & -\frac{1-\cos \left(\alpha_{k-1} T\right)}{\alpha_{k-1}} & 0 \\
0 & \cos \left(\alpha_{k-1} T\right) & 0 & -\sin \left(\alpha_{k-1} T\right) & 0 \\
0 & \frac{1-\cos \left(\alpha_{k-1} T\right)}{\alpha_{k-1}} & 1 & \frac{\sin \left(\alpha_{k-1} T\right)}{\alpha_{k-1}} & 0 \\
0 & \sin \left(\alpha_{k-1} T\right) & 0 & \cos \left(\alpha_{k-1} T\right) & 0 \\
0 & 0 & 0 & 0 & 1
\end{array}\right] \mathbf{x}_{k-1}} \\
+\left[\begin{array}{ccc}
\frac{T^{2}}{2} & 0 & 0 \\
T & 0 & 0 \\
0 & \frac{T^{2}}{2} & 0 \\
0 & T & 0 \\
0 & 0 & 1
\end{array}\right] \varepsilon_{k-1}
\end{gathered}
$$

Here, $T=1$ second (s) is the sampling interval, and $\varepsilon_{k-1}$ is the noise. In this paper, $\boldsymbol{\varepsilon}_{k-1}$ is defined by $\varepsilon_{k-1} \sim N\left(\varepsilon_{k-1} ; 0, \mathbf{Q}\right)$. $\mathbf{Q}$ is the covariance matrix, denoted by $\mathbf{Q}=$ $\operatorname{diag}\left(\sigma_{x, \boldsymbol{\varepsilon}}^{2}, \sigma_{y, \varepsilon}^{2}, \sigma_{\alpha}^{2}\right) . \sigma_{x, \boldsymbol{\varepsilon}}$ and $\sigma_{y, \varepsilon}$ are the errors. In this paper, we set $\sigma_{x, \varepsilon}=\sigma_{y, \varepsilon}=$ 1 meter $/$ second $^{2}\left(\mathrm{~m} / \mathrm{s}^{2}\right)$, and $\sigma_{\alpha}=\pi / 180 \mathrm{rad}$. The initial states, appearing times, and disappearing times of five targets are listed in Table 3.

Besides, we depict the observation model by

$$
\mathbf{z}_{k}=\left[\begin{array}{c}
\arctan \left(\frac{s_{k}^{y}}{s_{k}^{x}}\right) \\
\sqrt{\left(s_{k}^{x}\right)^{2}+\left(s_{k}^{x}\right)^{2}}
\end{array}\right]+\boldsymbol{\eta}_{k}
$$

where, $\boldsymbol{\eta}_{k}$, denoted by $\boldsymbol{\eta}_{k} \sim N\left(\boldsymbol{\eta}_{k} ; 0, \mathbf{R}\right)$, is the observation noise. $\mathbf{R}$ is the covariance. In this paper, $\sigma_{\theta}=\pi / 180 \mathrm{rad}$ and $\sigma_{r}=1$ meter $(\mathrm{m})$. In the detection region, the clutters are uniformly distributed. The angle and distance ranges of clutters are $(0, \pi / 2) \mathrm{rad}$ and $(0,1000) \mathrm{m}$, respectively. We demontrate the trajectories of the five targets in Figure 1.

For parameters, we set the gating threshold $T_{h}=16$ (the same as [28]). We also set the probability of survival $p_{s}\left(\mathbf{x}_{k}\right)=0.99$, and the probability of detection $p_{d}\left(\mathbf{x}_{k}\right)=0.95$, which are the same as [23]. All of the simulation results are achieve by a computer with i7 processor, 8GB RAM and MATLAB 2016a. 


\section{Comparison of Estimation Accuracy on Certain Number of Clutters}

Using parameters of Section, we implement ET-GM-PHD, CK-ET-GM-PHD and our ETSCI-GM-PHD approaches by certain number of clutters, where the number of clutters is set to be 20. Each approach is performed with 500 Monte Carlo runs. For justice, we use the adaptive method in Section to initiate the birth intensities of these three approaches. In addition, we use the extended Kalman filter to compute means and covariances of GM components of the ET-GM-PHD approach. The estimated trajectories of these approaches are plotted in Figure 2. From these figures, we can observe that the estimated points of these approaches are covered with the ground truth. In contrast to the ET-GM-PHD and CK-ETGM-PHD approaches, our ET-SCI-GM-PHD approach has the most number of covered points.

Furthermore, we compare the OSPA distances of these approaches in Figure 3. In contrast to the ET-GM-PHD and CK-ET-GM-PHD approaches, the curve of our ET-SCI-GM-PHD approach has smaller values. Notice that large OSPA distance denotes large tracking errors, the proposed ET-SCI-GM-PHD has smallest tracking errors in all of these approaches.

Furthermore, we also plot the estimated numbers and corresponding RMSEs of the three approaches in Figure 4, respectively. From Figure 4(a), we can observe that the estimated numbers of our ET-SCI-GM-PHD approach are most close to the ground truth, thus enjoying the smallest RMSE in Figure 4(b). Besides, we provide the numerical results of these approaches in Table 4. According to Table 4, the OSPA distance and RMSE of the ET-SCI-GM-PHD approach are the smallest in all of these approaches. Therefore, our ETSCI-GM-PHD approach can achieve better accuracy for estimating states and number of targets than the other two.

\section{Comparison of Estimation Accuracy on Various Numbers of Clutters}

For validating the effects of clutters on tracking accuracy, we change the number of clutters and perform the three approaches. Here, the clutter number is ranged from 5 to 50 . The OSPA distances and RMSEs of these approaches under different numbers of clutters are illustrated in Figure 5 and 6. Since the ET-GM-PHD approach uses the the first-order Taylor series to approximate the nonlinear function of (2), it cost larger tracking errors than the other two approaches (Seen in Figure 5 and 6). From these figures, we can also see that the curves of the proposed ET-SCI-GM-PHD approach has the smallest values in all of the three approach. Besides, when the number of clutters increases from 5 to 50 , the OSPA distances and RMSEs of these approaches are enhanced. However, the changes of the proposed approach in Figure 5 and 6 seems less than the other two. It is because that we apply the SCIF to estimate the means and covariances of GM component, and use the gating to decrease the effects of clutters. Thus, our ET-SCI-GM-PHD approach can achieve better tracking performance than the ET-GM-PHD and CK-ET-GM-PHD approaches.

\section{Comparison of Estimation Accuracy under different probabilities}

In this section, we implement the ET-GM-PHD, CK-ET-GM-PHD and our ET-SCI-GMPHD approaches using different probabilities of detection. Here, the probability of detection is set to be $0.85,0.90,0.95$, and 0.99 . The comparison of these approaches are run with 500 Monte Carlo. The clutter number is set to be 20. Table 5 demonstrates the OSPA distances and RMSEs of the three approaches.

In Table 5, when the probability ranges from 0.85 to 0.95 , the OSPA distances and RMSEs are rapidly decreased. Recall that when the probability of detection grows large, the 
observation has large probability to be generated by multi targets. Thus, the tracking errors can be reduced. When the probability of detection becomes 0.95 and 0.99 , the OSPA distances and RMSEs are slowly decreased. The estimated results of the three approaches becomes stable. In addition, owing to the SCIF and gating methods, our ET-SCI-GM-PHD approach has the least tracking errors in all of the three approaches, yields to the best tracking performance.

\section{Conclusion}

In this paper, we have proposed the ET-SCI-GM-PHD approach. Such an approach can be employed to estimate the states and number of multi extended targets. As for our approach, we compute the predicted mean and square-root factor of the covariance of the GM component by the SCKF method. Then, by propagating the information forms of the mean and square-root of covariance, the SCIF with gating method has been utilized for computing the posterior means and covariances of GM components. Thus, the conventional ET-GMPHD approach can be applied into nonlinear multi target tracking scenarios. In order to reduce the effect of clutters, we use a gating method in updating stage of our approach for obtaining the most possible cell. For initiating the birth intensity, an observation driven method has been present in this paper. In our method, we use the conditional probability to represent the association between the target and its corresponding cell. Based on such probability, we can approximate the most possible partition. Besides, the cells corresponding to the estimated states can also be obtained. Then, GM components of the birth intensity can be computed by removing these cells from the obtained partition. Thus, our approach can initiate the birth intensity adaptively. The simulation results shows that our ET-SCI-GMPHD approach outperforms the conventional ET-GM-PHD and CK-ET-GM-PHD under the metric of OSPA distance and RMSE.

This paper concentrates on improving the tracking performance of the conventional ETGM-PHD approach in nonlinear multi extended target tracking. It simply adopt the existed partition method of the ET-GM-PHD approach. Other clustering methods for partition grouping may be integrated in our approach for the future work. Otherwise, optimizing the parameters of partition grouping can considered as another direction.

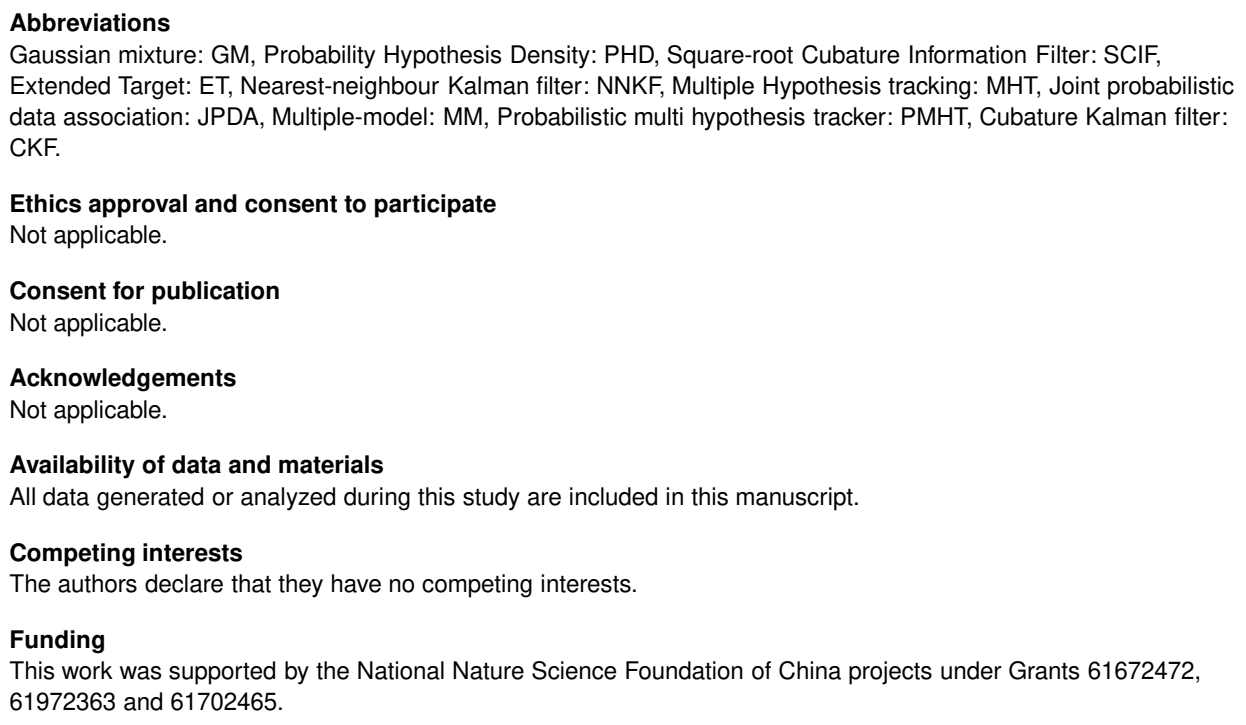




\section{Authors' contributions}

Methodology: Z.L. Validation: F.Y. and L.J. Formal analysis: Z.L. and X.Q. Investigation: F.Y. Writing original draft preparation: Z.L. and X.Q. The authors read and approved the manuscript.

\section{Authors' information}

School of Information and Communication Engineering, North University of China, Taiyuan, 030051, People's Republic of China

Zhe Liu, Fengbao Yang, Linna Ji and Xiqiang Qu

\section{Author details}

School of Information and Communication Engineering, North University of China, 030051 Taiyuan, China.

\section{References}

1. Feldmann, M., Franken, D., Koch, W.: Tracking of extended objects and group targets using random matrices. IEEE Transactions on Signal Processing 59(4), 1409-1420 (2008)

2. Ahmed, A.M., Alameer, A., Erni, D.: Maximizing information extraction of extended radar targets through mimo beamforming. IEEE Geoscience and Remote Sensing Letters 16(4), 539-543 (2019)

3. Liu, Z., Ji, L., Yang, F., Qu, X., Yang, Z., Qin, D.: Cubature information Gaussian mixture probability hypothesis density approach for multi extended target tracking. IEEE Access (7), 103678-103692 (2019)

4. Yoon, J.H., Kim, D.Y., Bae, S.H., Shin, V.: Joint initialization and tracking of multiple moving objects using doppler information. IEEE Transactions on Signal Processing 59(7), 3447-3452 (2011)

5. Zhe, L., Wang, Z., Mai, X., Lan, Y., Liu, J.: A novel CIF-based SMC-PHD approach for tracking multiple nonlinear targets. In: Proceedings of IEEE Radar Conference (RadarCon), pp. 555-560 (2015). IEEE

6. Feldmann, M., Franken, D., Koch, W.: Tracking of extended objects and group targets using random matrices. IEEE Transactions on Signal Processing 59(4), 1409-1420 (2011)

7. Lan, J., Li, X.R.: Tracking of maneuvering non-ellipsoidal extended object or target group using random matrix. IEEE Transactions on Signal Processing 62(9), 2450-2463 (2014)

8. Tang, X., Li, M., Tharmarasa, R., Kirubarajan, T.: Seamless tracking of apparent point and extended targets using Gaussian process PMHT. IEEE Transactions on Signal Processing 67(18), 4825-4838 (2019)

9. Daniyan, A., Lambotharan, S., Deligiannis, A., Gong, Y., Chen, W.-H.: Bayesian multiple extended target tracking using labeled random finite sets and splines. IEEE Transactions on Signal Processing 66(22), 6076-6091 (2018)

10. Gilholm, K., Godsill, S., Maskell, S., Salmond, D.: Poisson models for extended target and group tracking. In: Proceedings of SPIE, pp. 5913001-5913012 (2005). SPIE

11. Mahler, R.: PHD filters for nonstandard targets, I: Extended targets. In: Information Fusion, 2009. FUSlon'09. 12th International Conference On, pp. 915-921 (2009). IEEE

12. Zhang, Y., Ji, H.: A robust and fast partitioning algorithm for extended target tracking using a Gaussian inverse wishart PHD filter. Knowledge-Based Systems 95, 125-141 (2016)

13. Zhang, Y., Ji, H., Hu, Q.: A box-particle implementation of standard PHD filter for extended target tracking. Information Fusion 34, 55-69 (2017)

14. Granström, K., Lundquist, C., Orguner, U.: A Gaussian Mixture PHD filter for extended target tracking. In: Proceedings of the International Conference on Information Fusion

15. Granström, K., Orguner, U., Mahler, R., Lundquist, C.: Corrections on:"extended target tracking using a gaussian-mixture phd filter". IEEE Transactions on Aerospace and Electronic Systems 53(2), 1055-1058 (2017)

16. Yan, B., Xu, N., Xu, L., Li, M.Q., Cheng, P.: An improved partitioning algorithm based on FCM algorithm for extended target tracking in PHD filter. Digital Signal Processing 90, 54-70 (2019)

17. Chen, J., Wang, N., Ma, L., Xu, B.: Extended target probability hypothesis density filter based on cubature Kalman filter. IET Radar, Sonar \& Navigation 9(3), 324-332 (2015)

18. Wu, J., Li, K., Zhang, Q., An, W., Jiang, Y., Ping, X., Chen, P.: Iterative RANSAC based adaptive birth intensity estimation in GM-PHD filter for multi-target tracking. Signal Processing 131, 412-421 (2017)

19. Liu, Z., Wang, Z., Xu, M.: Cubature information SMC-PHD for multi-target tracking. Sensors 16(5), 653 (2016)

20. Ristic, B., Clark, D., Vo, B.-N., Vo, B.-T.: Adaptive target birth intensity for phd and cphd filters. IEEE Transactions on Aerospace and Electronic Systems 48(2), 1656-1668 (2012)

21. Peng, C., Ye, W.: An improved adaptive et-phd algorithm for newborn target intensity. In: 2018 IEEE 3rd Advanced Information Technology, Electronic and Automation Control Conference (IAEAC), pp. 2137-2142 (2018). IEEE

22. Chandra, K.P.B., Gu, D.W.., I.Postlethwaite: Square root cubature information filter. IEEE Sensors Journal 13(2), 750-758 (2013)

23. Granstrom, K., Lundquist, C., Orguner, O.: Extended target tracking using a Gaussian-mixture PHD filter. IEEE Transactions on Aerospace and Electronic Systems 48(4), 3268-3286 (2012)

24. Arasaratnam, I., Haykin, S.: Cubature kalman filters. IEEE Transactions on Automatic Control 54(6), 1254-1269 (2009)

25. Mo, L., Song, X., Zhou, Y., Kang, S., Bar-Shalom, Y.: Unbiased converted measurements for tracking. IEEE Transactions on Aerospace and Electronic Systems 34(3), 1023-1027 (1998)

26. Schuhmacher, D., Vo, B.T., Vo, B.N.: A consistent metric for performance evaluation of multi-object filters. IEEE Transactions on Signal Processing 56(8), 3447-3457 (2008)

27. Vo, B.N., Ma, W.K.: The gaussian mixture probability hypothesis density filter. IEEE Transactions on Signal Processing 54(11), 4091-4104 (2006)

28. Yoon, J.H., Kim, D.U., Yoon, K.J.: Gaussian mixture importance sampling function for unscented SMC-PHD filter. Signal Processing 93(9), 2664-2670 (2013) 
[width $=0.7 /$ textwidth]Fig/Truetrac.eps

Figure 1 Ground-truth trajectories of five targets with the clutter number setting to be 20 . The target trajectories are depicted by circle-solid lines with different colors, while the asterisks denote clutters.

[width=1.9in]Fig/esttrac20EK.eps [width=1.9in]Fig/esttrac20SC.eps [width=1.9in]Fig/esttrac20SCl.eps

(a) The ET-GM-PHD approach. $\quad$ (b) The CK-EPHD approach. $\quad$ (c) The ET-SCI-GM-PHD approach.

Figure 2 Estimated trajectories of the three approaches with clutter number being 20 . We use the red (light) points to represent the estimated trajectories, while the the true trajectories denote the black (dark) solid lines. (a) The ET-GM-PHD approach; (b) The CK-EPHD approach; (c) The ET-SCI-GM-PHD approach.

\section{[width=0.7/textwidth]Fig/OSPAnon.eps}

Figure 3 OSPA distances of the three approaches with clutter number being 20.

[width=2in]Fig/MeanNum.eps [width=2in]Fig/RMSECom.eps
(a) Estimated numbers.
(b) RMSEs.

Figure 4 Estimated numbers and RMSEs of the three approaches with clutter number being 20. (a) Estimated numbers; (b) RMSEs.

$$
\text { [width=0.7/textwidth]Fig/OSPAClu.eps }
$$

Figure 5 Averaged OSPA distances of the ET-GM-PHD, CK-EPHD and our ET-SCI-GM-PHD approaches along with the clutter number changing from 5 to 50.

[width=0.7/textwidth]Fig/STDClu.eps

Figure 6 Averaged RMSEs of estimated number of the ET-GM-PHD, CK-EPHD and our ET-SCI-GM-PHD approaches along with the clutter number changing from 5 to 50 .

Tables

Table 1 Notation list

$\mathbf{C}_{i} \quad$ The $i$-th partition of the current observation set

$\mathbf{X}_{k} \quad$ The state set of multi-target at time $k$

$\mathbf{Z}_{k} \quad$ The observation set of multi-target at time $k$

$\mathbf{x}_{k} \quad$ The state of a dynamic target at time $k$

$\mathbf{z}_{k} \quad$ The observation of a dynamic target at time $k$

$N(\cdot) \quad$ The function of the Gaussian distribution

$\gamma_{k}(\cdot) \quad$ The birth intensity of target at time $k$

$p_{s}(\cdot) \quad$ The survival probability of target

$p_{d}(\cdot) \quad$ The detected probability of target

$D(\cdot \mid \cdot) \quad$ The intensity of multi-target 
Table 2 The ET-SCI-GM-PHD approach from time step $k-1$ to $k$

- Input: Birth component set $\mathbf{B}_{k-1}$, survival component set $\left\{x_{k-1}^{(i)}, \mathbf{P}_{k-1}^{(i)}, w_{k-1}^{(i)}\right\}_{i=1}^{L_{k-1}}$, and current observation set $\mathbf{Z}_{k}$

- Output: Target number $N_{k}$, birth component set $\mathbf{B}_{k}$, and estimated state set $\mathbf{X}_{k}^{e}$

- Compute the candidate partition set $\left\{\mathbf{C}_{l}\right\}_{l=1}^{N_{d}}$ of the current observation set $\mathbf{Z}_{k}$ by Table I in [23], where $N_{d}$ denotes the number of partitions, and $\mathbf{C}_{l}$ is the $l$-th partition.

- Approximate the predicted means and square-root factor of covariances $\left\{\mathbf{m}_{k \mid k-1}^{j}, \mathbf{S}_{k \mid k-1}^{j}\right\}$ of GM components by (29) and (30).

- Substitute $\left\{\mathbf{m}_{k \mid k-1}^{j}, \mathbf{P}_{k \mid k-1}^{j}\right\}$ into (10) to obtain the survival intensity $v_{s, k \mid k-1}(\mathbf{x})$, where $\mathbf{P}_{k \mid k-1}^{j}=$ $\mathbf{S}_{k \mid k-1}^{j}\left(\mathbf{S}_{k \mid k-1}^{j}\right)^{T}$.

- Construct the birth intensity $\gamma_{k}(\mathbf{x})$, and obtain the predicted intensity $D_{k \mid k-1}(\mathbf{x})$ of (9) using $\gamma_{k}(\mathbf{x})$ and $v_{s, k \mid k-1}(\mathbf{x})$.

- Extract the most possible cell $\mathbf{W}_{c}$ from $\tilde{\mathbf{C}}_{k}$ by (38) and (39).

- Evaluate the information forms $\mathbf{y}_{k \mid k-1}^{j}$ and $\mathbf{Y}_{s, k \mid k-1}$ corresponding to $\mathbf{m}_{k \mid k-1}^{j}$ and $\mathbf{S}_{k \mid k-1}^{j}$ by (40) and (47).

- Calculate the square-root fact $\mathbf{Y}_{I, k}^{j}$ and state contribution $\mathbf{i}_{k}^{j}$ by (50) and (53).

- Compute $\mathbf{y}_{k}$ and $\mathbf{Y}_{s, k}^{j}$ by (45) and (55).

- Obtain $\mathbf{m}_{k}^{j}$ and $\mathbf{P}_{k}^{j}$ by $\mathbf{y}_{k}^{j}$ and $\mathbf{Y}_{s, k}^{j}$ using (40) and (47), where $\mathbf{P}_{k}^{j}=\mathbf{S}_{k}^{j}\left(\mathbf{S}_{k}^{j}\right)^{T}$.

- Approximate the detected intensity by (16)-(19) with computed component $\left\{\mathbf{m}_{k}^{j}, \mathbf{P}_{k}^{j}\right\}$.

- Obtain the updated intensity $D_{k \mid k}^{D}(\mathbf{x})$ by (12).

- Prune the GM components of $D_{k \mid k}^{D}(\mathbf{x})$ using the pruning method of [27]. Here, we consider the remaining components $\left\{x_{k}^{(i)}, \mathbf{P}_{k}^{(i)}, w_{k}^{(i)}\right\}_{i=1}^{L_{k}}$ as the survival components at next time step, where $L_{k}$ refers to the number of GM components after pruning.

- Estimate the state set $\mathbf{X}_{k}^{e}$, and achieve the target number $N_{k}$.

- Approximate the candidate partition $\tilde{\mathbf{C}}_{k}$, and obtain the cell set $\tilde{\mathbf{C}}_{k}^{b}$ by (62) and (63).

- Calculate the birth component set $\mathbf{B}_{k}=\left\{\mathbf{m}_{k, i}^{b}, \mathbf{P}_{k, i}^{b}\right\}_{i=1}^{N_{k}, b}$ by (65)-(67).

- Return $N_{k}, \mathbf{X}_{k}^{e}$ and $\mathbf{B}_{k}$.

Table 3 Initial states of targets.

\begin{tabular}{cccc}
\hline Target & State & Appearing(s) & Disappearing(s) \\
\hline 1 & {$[320,5,320,5,0]$} & 1 & 40 \\
2 & {$[400,-5,400,5,0]$} & 8 & 50 \\
3 & {$[375,5,375,-5,0]$} & 25 & 70 \\
4 & {$[400,5,325,-5,0]$} & 59 & 70 \\
5 & {$[325,-5,375,5,0]$} & 59 & 70 \\
\hline
\end{tabular}

Table 4 Averaged Estimation Errors.

\begin{tabular}{ccc}
\hline Approach & OSPA $(\mathbf{m})$ & RMSE \\
\hline ET-GM-PHD & 29.23 & 0.43 \\
CK-PHD & 17.29 & 0.25 \\
ET-SCI-GM-PHD & 16.19 & 0.17 \\
\hline
\end{tabular}

Table 5 Tracking Performance over different detection probabilities.

\begin{tabular}{ccccccccc}
\hline & \multicolumn{3}{c}{$\boldsymbol{p}_{\boldsymbol{d}}=\mathbf{0 . 8 5}$} & & \multicolumn{3}{c}{$\boldsymbol{p}_{\boldsymbol{d}}=\mathbf{0 . 9 0}$} \\
\cline { 2 - 4 } \cline { 7 - 8 } & ET-GM-PHD & CK-EPHD & ET-SCI-GM-PHD & & ET-GM-PHD & CK-EPHD & ET-SCI-GM-PHD \\
\hline OSPA(m) & 35.80 & 21.09 & 18.49 & & 29.56 & 18.77 & 16.54 \\
RMSE & 0.54 & 0.44 & 0.22 & & 0.44 & 0.31 & 0.18 \\
\hline & \multicolumn{3}{c}{$\boldsymbol{p}_{\boldsymbol{d}}=\mathbf{0 . 9 5}$} & & \multicolumn{3}{c}{$\boldsymbol{p}_{\boldsymbol{d}}=\mathbf{0 . 9 9}$} \\
\cline { 2 - 4 } \cline { 7 - 8 } & ET-GM-PHD & CK-EPHD & ET-SCI-GM-PHD & & ET-GM-PHD & CK-EPHD & ET-SCI-GM-PHD \\
\hline OSPA(m) & 28.25 & 17.13 & 15.93 & & 28.13 & 16.59 & 15.84 \\
RMSE & 0.42 & 0.29 & 0.16 & & 0.41 & 0.28 & 0.16 \\
\hline
\end{tabular}


Figures

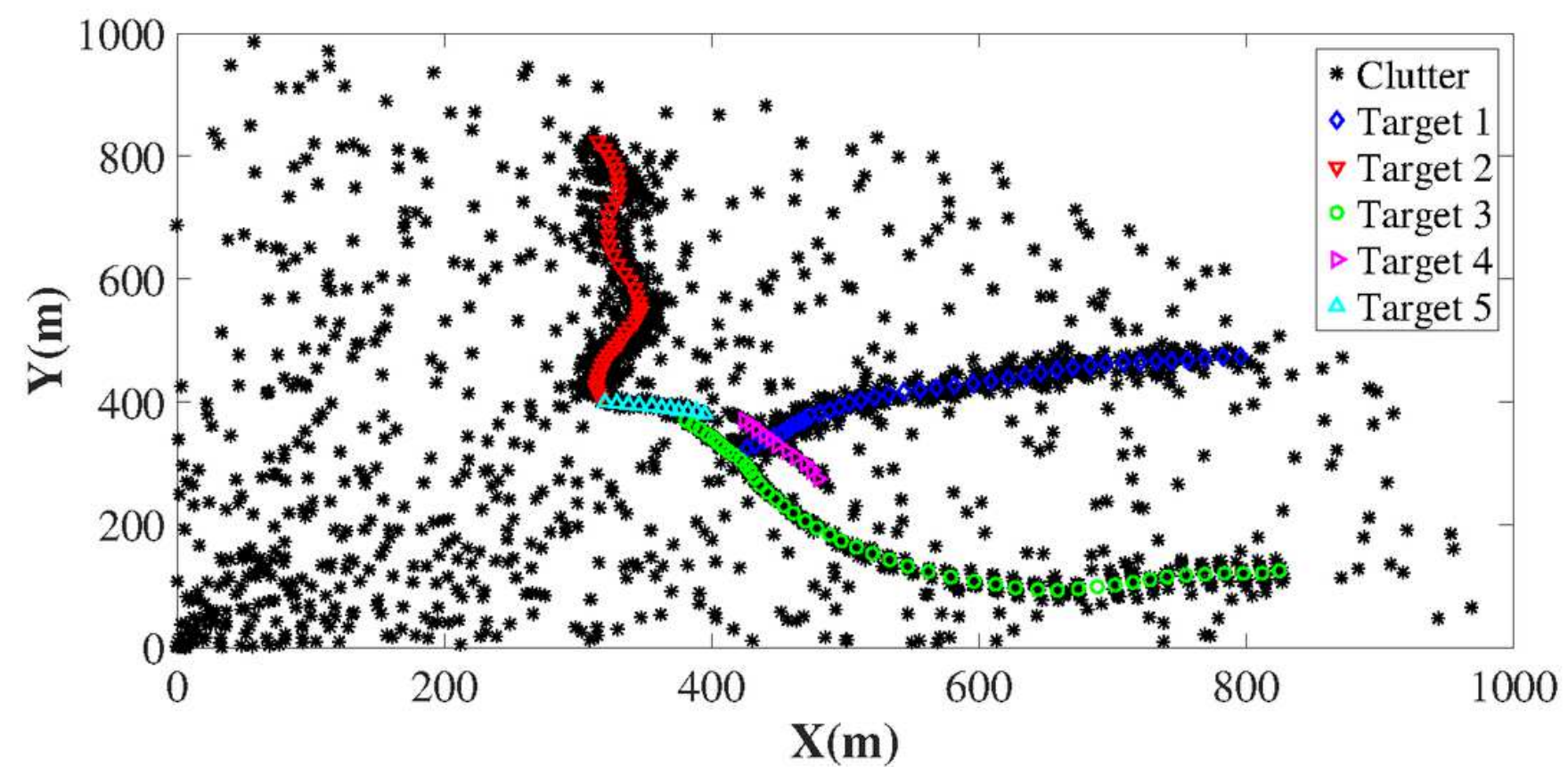

Figure 1

Ground-truth trajectories of five targets with the clutter number setting to be 20 . The target trajectories are depicted by circle-solid lines with different colors, while the asterisks denote clutters. 
a

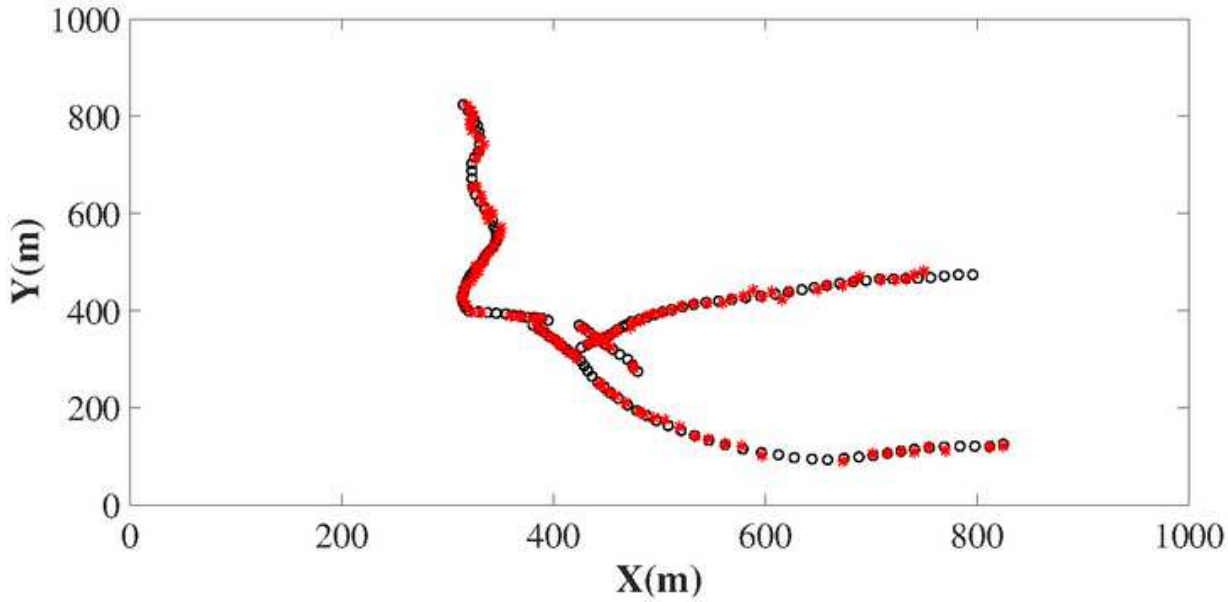

b
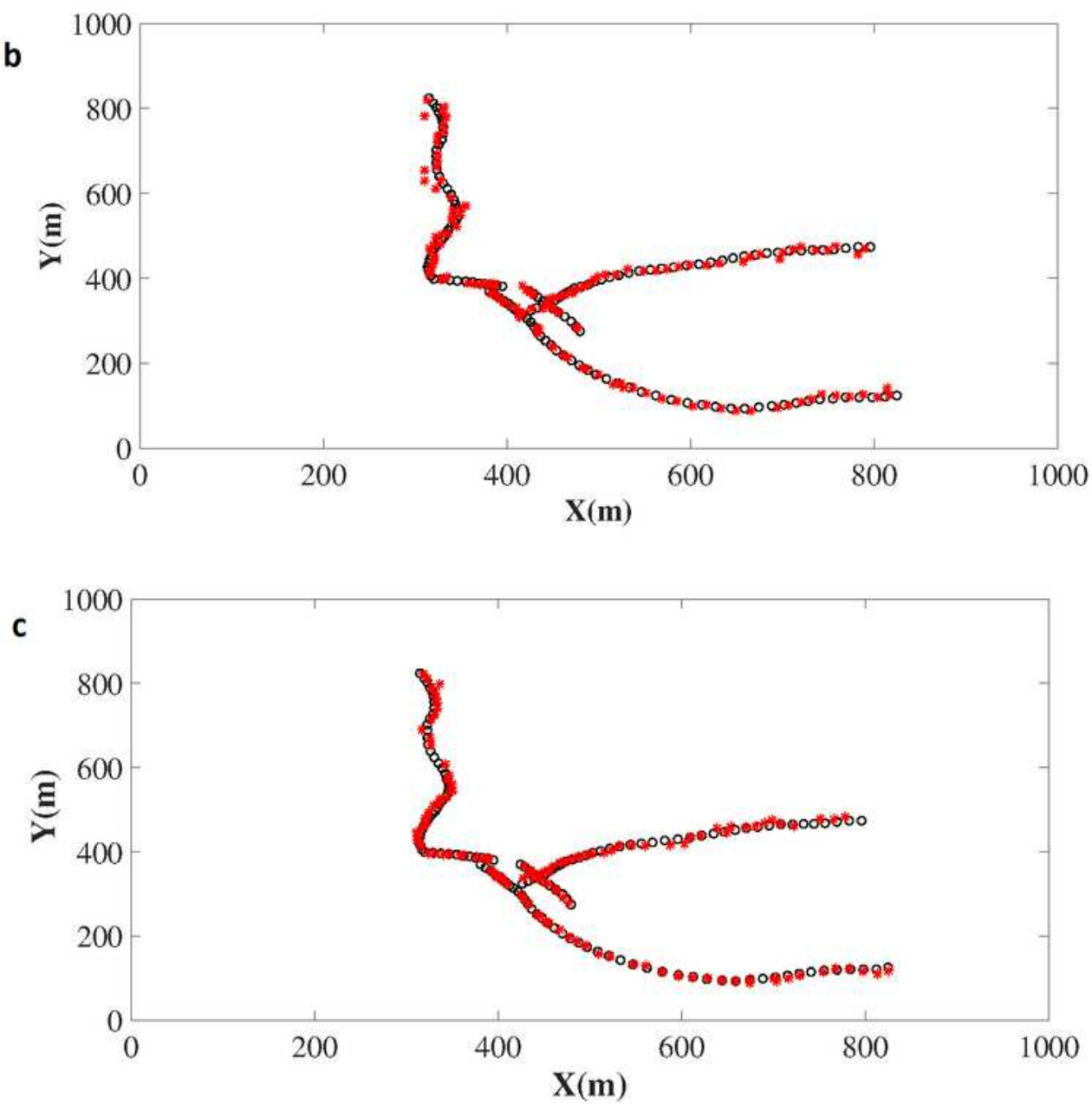

Figure 2

Estimated trajectories of the three approaches with clutter number being 20. We use the red (light) points to represent the estimated trajectories, while the the true trajectories denote the black (dark) solid lines.

(a) The ET-GM-PHD approach; (b) The CK-EPHD approach; (c) The ET-SCI-GM-PHD approach. 


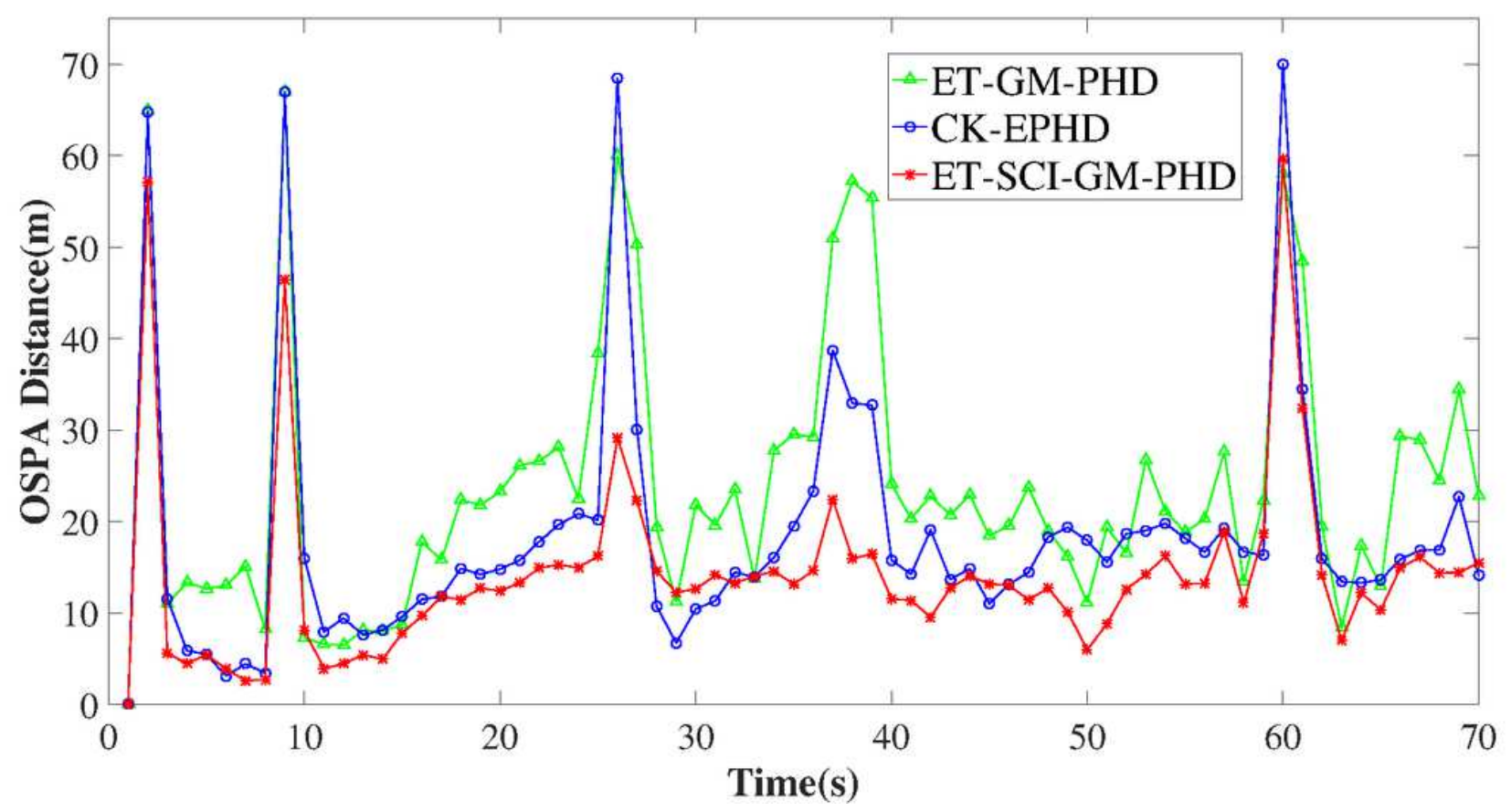

Figure 3

OSPA distances of the three approaches with clutter number being 20 . 

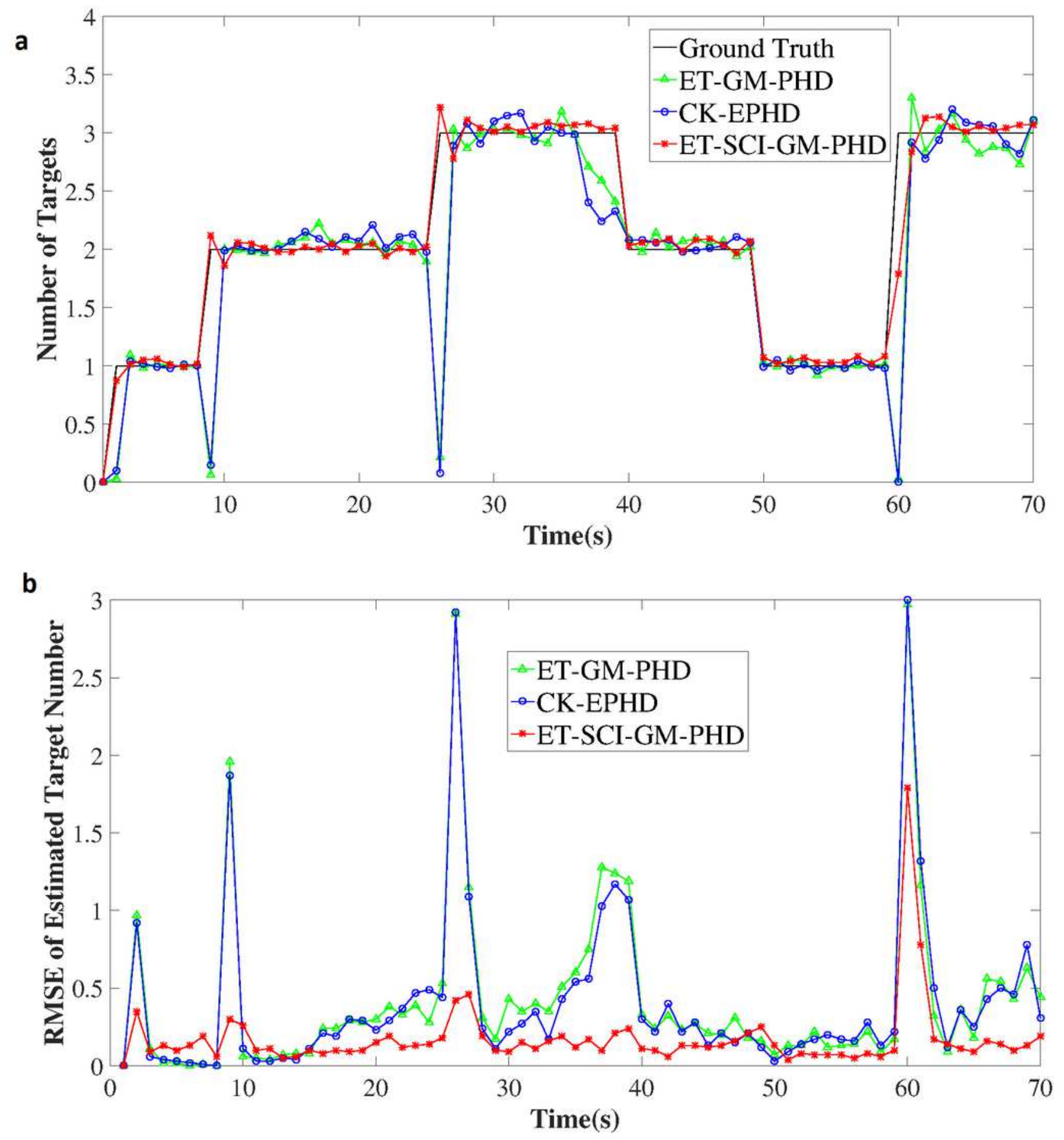

Figure 4

Estimated numbers and RMSEs of the three approaches with clutter number being 20. (a) Estimated numbers; (b) RMSEs. 


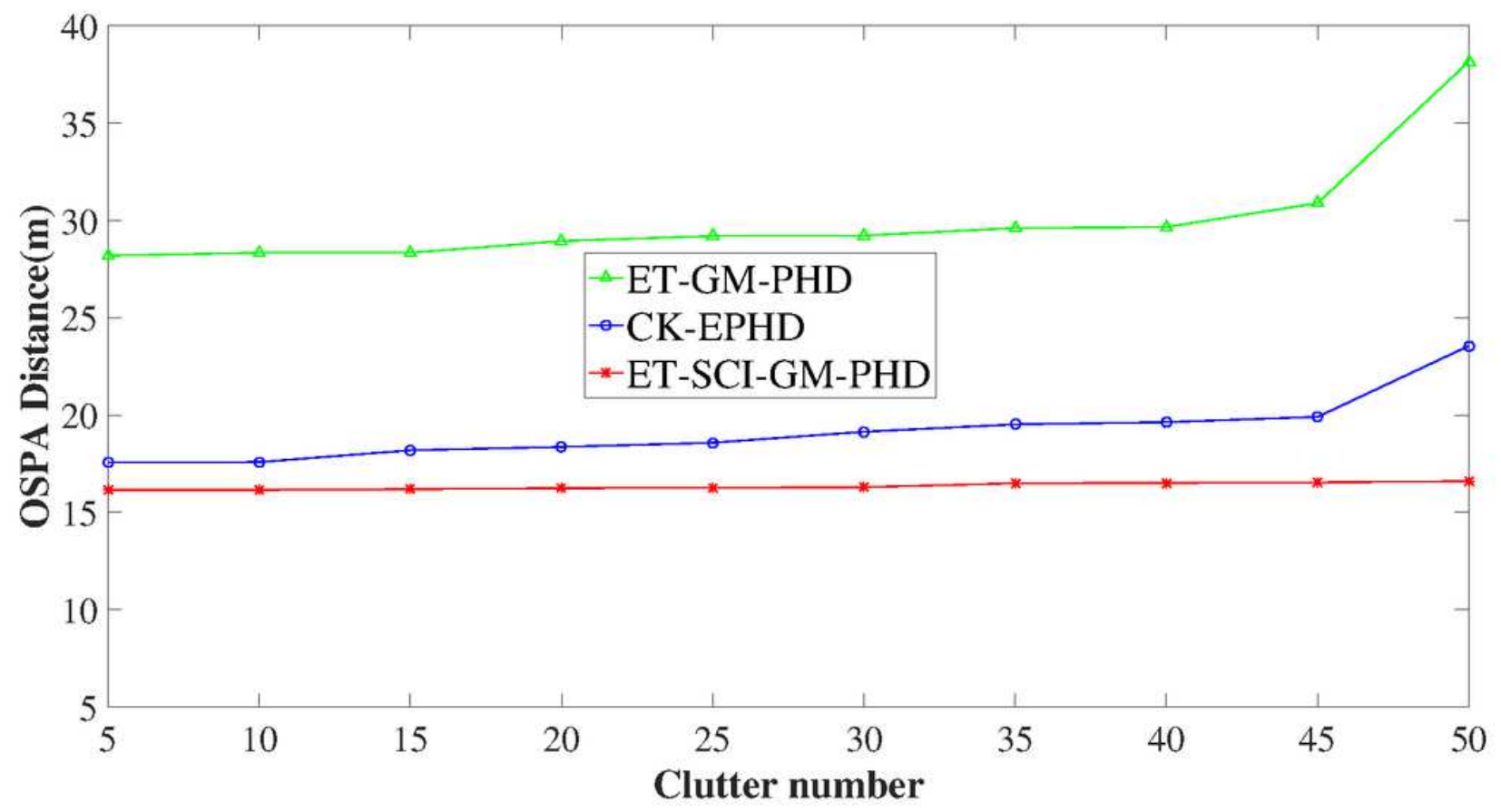

Figure 5

Averaged OSPA distances of the ET-GM-PHD, CK-EPHD and our ET-SCI-GM-PHD approaches along with the clutter number changing from 5 to 50 .

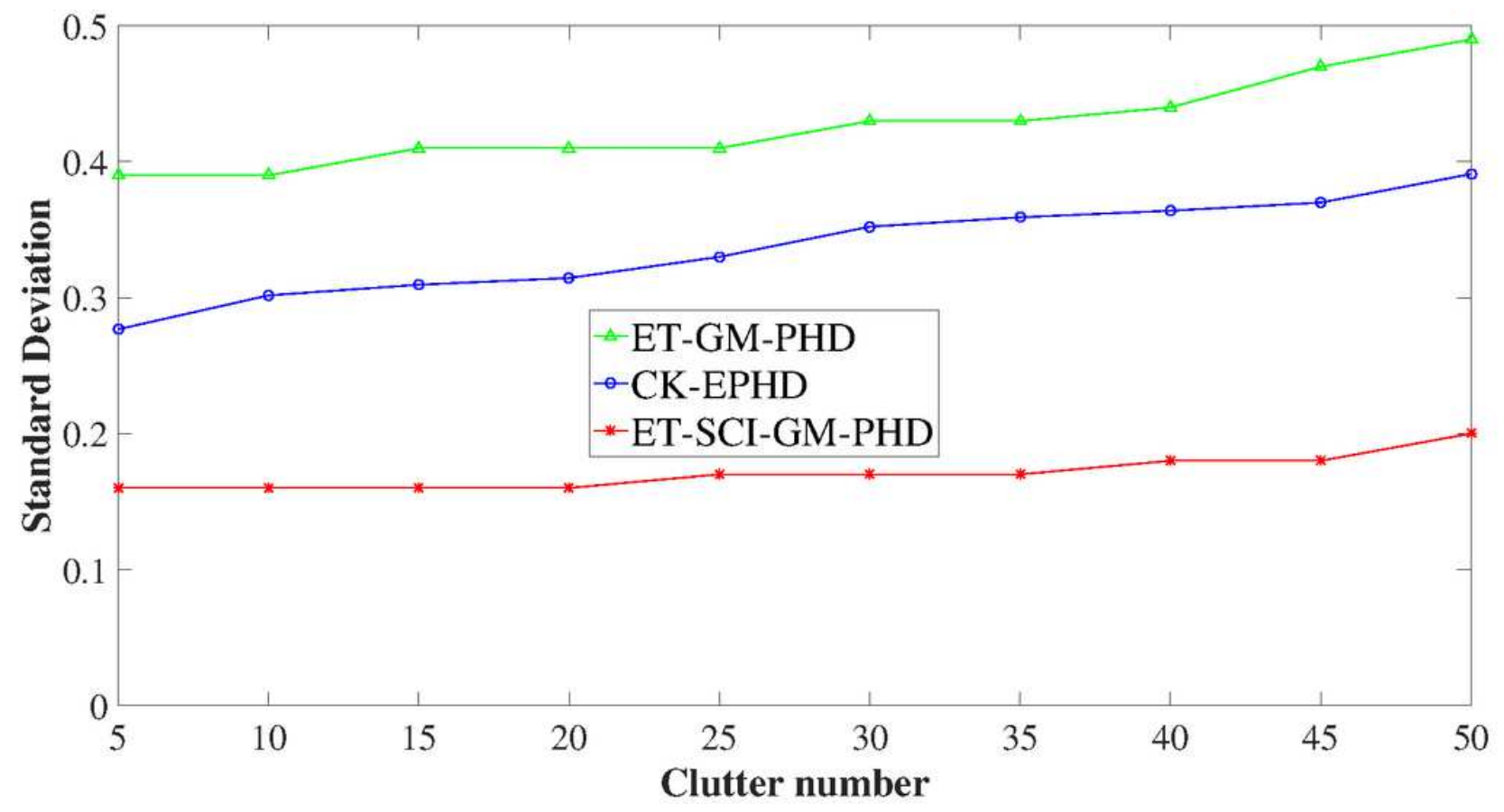

Figure 6

Averaged RMSEs of estimated number of the ET-GM-PHD, CK-EPHD and our ET-SCI-GM-PHD approaches along with the clutter number changing from 5 to 50 . 